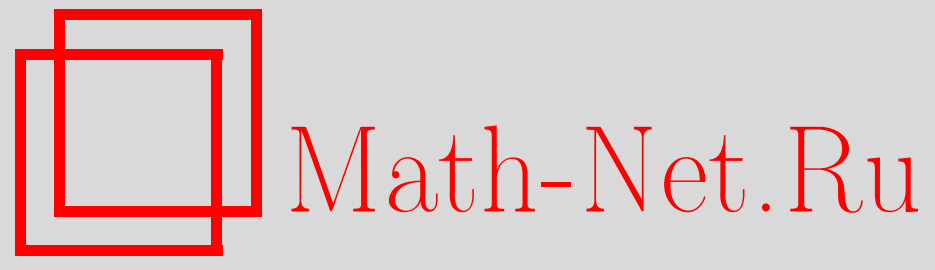

Ю. С. Мишура, Г. М. Шевченко, Аппроксимационные схемы для стохастических дифференциальных уравнений в гильбертовом пространстве, Теория вероятн. и ее примен., 2006, том 51, выпуск 3, 476495

DOI: https://doi.org/10.4213/tvp35

Использование Общероссийского математического портала MathNet.Ru подразумевает, что вы прочитали и согласны с пользовательским соглашением

http://www.mathnet.ru/rus/agreement

Параметры загрузки:

IP : 52.23 .180 .231

26 апреля 2023 г., 10:09:08

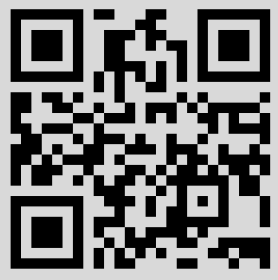


(C) 2006 г. МИШУРА Ю. С.*, ШЕВЧЕНКО Г. М.*

\title{
АППРОКСИМАЦИОННЫЕ СХЕМЫ ДЛЯ СТОХАСТИЧЕСКИХ ДИФФЕРЕНЦИАЛЬНЫХ УРАВНЕНИЙ В ГИЛЬБЕРТОВОМ ПРОСТРАНСТВЕ ${ }^{1)}$
}

\begin{abstract}
Для решений уравнений Ито-Вольтерра и полулинейных уравнений эволюционного типа в гильбертовом пространстве рассматриваются аппроксимации по схеме Мильштейна, аппроксимации конечномерными процессами и аппроксимации решениями стохастических дифференциальных уравнений с ограниченными коэффициентами. Для конечномерных аппроксимаций доказана сходимость в среднем квадратическом, в остальных двух случаях получены результаты о скорости сходимости аппроксимаций в среднем квадратическом.
\end{abstract}

Ключевые слова и фразы: стохастические дифференциальные уравнения в гильбертовом пространстве, аппроксимации с дискретным временем, схема Мильштейна, уравнение типа ИтоВольтерра.

\section{Введение}

Приближенное решение стохастических дифференциальных уравнений (СДУ) имеет широкие применения. Классический пример такого применения основан на формуле Фейнмана-Каца, связывающей решение параболического уравнения в частных производных с решением СДУ. Много уравнений, возникающих при моделировании физических, химических, биологических явлений, котировок акций, содержат случайность. Не всегда, впрочем, эта случайность адекватно моделируется классическим белым шумом - винеровским процессом, но часто при выборе надлежащего масштаба или при рассмотрении асимптотического поведения системы случайность становится гауссовской.

Идея приближенного решения СДУ методом, аналогичным методу ломаных Эйлера для неслучайных уравнений, возникает в статье Г. Маруямы [13]. Дальнейшее развитие теории связано с работами

* Киевский национальный университет им. Тараса Шевченко, механико-математический факультет, ул. Владимирская, 64, 01033 Киев, Украина; е-mail: myus@univ.kiev.ua; zhora@univ.kiev.ua

1) Второй автор поддержан INTAS (грант YSF 03-55-2447). 
Г.Н. Мильштейна [23], построившего схему большего порядка точности, и В. Вагнера и Э. Платена [19], предложивших метод построения схем высшего порядка точности с помощью стохастических разложений Тейлора. Монографии [24], [12] содержат практически полную теорию аппроксимации конечных систем СДУ с регулярными коэффициентами. Также хочется отметить работу [17], где приводится близкая к исчерпывающей (на момент издания) библиография по численному решению СДУ, и монографию [22], где, кроме обширной теории решения конечных систем СДУ, приведен новый (по сравнению с [24], [12]) метод моделирования кратных интегралов от винеровского процесса. Отметим еще статью [11], где доказана предельная теорема (без скорости сходимости) для конечномерных уравнений Ито-Вольтерра. Близкими по тематике к данной статье являются работы по аппроксимации стохастических дифференциальных уравнений в частных производных (СДУЧП). Это, в частности, статьи И. Дендя и Н. В. Крылова [6], [7], в которых оценивается скорость сходимости аппроксимаций СДУЧП методом «splittinguр», А. Миле и М. Санс-Соле [14], рассмотревших аппроксимации стохастического уравнения распространения волн, И. Дендя и А. Миле [8], рассмотревших приближенное решение уравнений с монотонными операторами, К. Ду и Т. Чжан [4], сделавших оценку скорости сходимости аппроксимаций линейных эллиптических и параболических уравнений, Т. Шардлоу [18], Р. Петтерсона и М. Сигнала [15], изучивших аппроксимации для стохастического уравнения теплопроводности, и диссертации Л. Романа [16], Ю. Яна [20], исследовавших схемы типа Рунге-Кутта для параболических СДУЧП.

Данная работа является в определенном смысле продолжением работы [25], где для полулинейных уравнений с неограниченным сносом и уравнений типа Ито-Вольтерра в гильбертовом пространстве обобщен классический результат о скорости сходимости аппроксимаций Эйлера. В разделе 1 получены результаты о скорости сходимости аппроксимаций по схеме Мильштейна для уравнений типа Ито-Вольтерра и полулинейных уравнений эволюционного типа. Оказывается, что скорость сходимости такая же, как для систем обычных СДУ. В разделе 2 для тех же типов уравнений доказана сходимость в среднем квадратическом конечномерных аппроксимаций. В разделе 3 рассматривается аппроксимация решений полулинейных уравнений с неограниченным оператором решениями уравнений с ограниченным оператором. Получен результат о скорости сходимости в среднем квадратическом таких аппроксимаций.

\section{1. Приближение решений уравнений по схеме Мильштейна}

Пусть $X$ - сепарабельное гильбертово пространство, $(\Omega, \mathscr{F}, \mathbf{P})-$ вероятностное пространство, $\left(\mathscr{F}_{t}, t \in[0, T]\right)-$ поток $\sigma$-алгебр, $W(t)-$ $\mathscr{F}_{t}$-согласованный цилиндрический винеровский процесс в $X$. 
Рассмотрим стохастическое эволюционное уравнение

$$
X(t)=X_{0}+\int_{0}^{t}(A X(s)+a(s, X(s))) d s+\int_{0}^{t} b(s, X(s)) d W(s) .
$$

Здесь $a$ и $b$ - измеримые отображения из $[0, T] \times X$ в $X$ и $\mathscr{L}_{2}(X, X)($ пространство операторов Гильберта-Шмидта) соответственно, $A: D(A) \rightarrow$ $X$ - линейный, вообще говоря неограниченный, оператор, $X_{0}-\mathscr{F}_{0}$-измеримая интегрируемая с квадратом случайная величина, принадлежащая $D(A)$ п.н.

В дальнейшем мы будем опускать индекс у нормы $\|\cdot\|_{X}$ и писать просто $\mathscr{L}_{2}$ вместо $\mathscr{L}_{2}(X, X)$, а также $\mathscr{L}$ вместо $\mathscr{L}(X, X)$, где $\mathscr{L}(X, Y)-$ пространство линейных непрерывных операторов из $X$ в $Y$. Bсе несущественные постоянные будем обозначать $C$.

В работе [25] рассмотрены аппроксимации по схеме Эйлера для таких уравнений. Общий подход для схемы Мильштейна такой же. Предположим, что $A$ порождает сильно непрерывную полугруппу $\{U(t), 0 \leqslant t \leqslant T\}$. Тогда если существует сильное решение уравнения (1.1), то оно также является «мягким», т.е. удовлетворяет уравнению

$$
X(t)=U(t) X_{0}+\int_{0}^{t} U(t-s) a(s, X(s)) d s+\int_{0}^{t} U(t-s) b(s, X(s)) d W(s)
$$

Последнее уравнение является частным случаем уравнения типа ИтоВольтерра, научившись приближать которое, можно применить полученные результаты для стохастических эволюционных уравнений.

1.1. Приближенное решение уравнений типа Ито-Вольтерра по схеме Мильштейна. Абстрактное уравнение ИтоВольтерра имеет вид

$$
X(t)=m(t)+\int_{0}^{t} a(t, s, X(s)) d s+\int_{0}^{t} b(t, s, X(s)) d W(s), \quad t \in[0, T]
$$

где $a: S \times X \rightarrow X, b: S \times X \rightarrow \mathscr{L}_{2}$ - измеримые отображения $(S=$ $\left.\left\{(t, s) \in[0, T]^{2}: s \leqslant t\right\}\right), m(t)$ - некоторый $\mathscr{F}_{t}$-согласованный непрерывный квадратично интегрируемый процесс. Как и в случае обычных СДУ, условия липшицевости и линейного роста

$$
\begin{gathered}
\|a(t, s, x)-a(t, s, y)\|+\|b(t, s, x)-b(t, s, y)\|_{\mathscr{L}_{2}} \leqslant C\|x-y\| \\
\|a(t, s, x)\|+\|b(t, s, x)\|_{\mathscr{L}_{2}} \leqslant C(1+\|x\|)
\end{gathered}
$$

гарантируют, что непрерывное потраекторно единственное решение уравнения (1.3) существует в $L_{2}(\Omega)$, причем sup $\mathbf{E}\|X(t)\|^{2}<\infty$ (см., например, [21]). Если коэффициенты $a, b$ дифференцируемы по первой 
переменной, производные $a_{t}^{\prime}, b_{t}^{\prime}$ удовлетворяют условиям линейного роста и процесс $m(t)$ имеет стохастический дифференциал, то, применяя стохастическую теорему Фубини, получаем, что процесс $X(t)$ имеет стохастический дифференциал

$$
\begin{aligned}
d X(t)=d m(t)+( & a(t, t, X(t))+\int_{0}^{t} a_{t}^{\prime}(t, s, X(s)) d s \\
& \left.+\int_{0}^{t} b_{t}^{\prime}(t, s, X(s)) d W(s)\right) d t+b(t, t, X(t)) d W(t) .
\end{aligned}
$$

Предположим, что производная по Фреше $\frac{\partial}{\partial x} b=b_{x}^{\prime}$ существует в $\mathscr{L}\left(X, \mathscr{L}_{2}\right)$ и является измеримой ограниченной функцией всех своих аргументов. Построим аппроксимации уравнения (1.3) по схеме Мильштейна [24]. Для заданного числа $N \in \mathrm{N}$ положим $\delta=T / N$ и возьмем $\tau_{n}=n \delta, n=0,1, \ldots, N$, - равномерное разбиение отрезка $[0, T]$. Предполагая, что мы имеем некоторое приближение $m^{\delta}(t)$ процесса $m(t)$, аппроксимации в узлах разбиения определим последовательно:

$$
\begin{array}{r}
Y_{n+1}^{\delta}=m^{\delta}\left(\tau_{n+1}\right)+\sum_{i=0}^{n}\left(a\left(\tau_{n+1}, \tau_{i}, Y_{i}^{\delta}\right) \delta+b\left(\tau_{n+1}, \tau_{i}, Y_{i}^{\delta}\right)\left(W\left(\tau_{i+1}\right)-W\left(\tau_{i}\right)\right)\right. \\
\left.\quad+\int_{\tau_{i}}^{\tau_{i+1}} b_{x}^{\prime}\left(\tau_{n+1}, \tau_{i}, Y_{i}^{\delta}\right) b\left(\tau_{i}, \tau_{i}, Y_{i}^{\delta}\right)\left(W(s)-W\left(\tau_{i}\right)\right) d W(s)\right) .(1.5)
\end{array}
$$

3 а м е ч а н и е 1.1. Обратим внимание, что в формуле (1.5) появляются интегралы от винеровского процесса, распределение которых довольно сложно моделировать. Возникает естественный вопрос: а нельзя ли получить ту же скорость сходимости для иной схемы, в которой участвовали бы только приращения винеровского процесса? Ответ на этот вопрос дает известный «парадокс Кларка-Камерона» (см., например, [3]): при размерности больше 1 любые аппроксимации, зависящие только от приращений винеровского процесса на отрезках разбиения, имеют, вообще говоря, такой же порядок сходимости, что и схема Эйлера.

З а м е ч а н и е 1.2. Интегралы в (1.5) определены корректно, если

$$
\int_{\tau_{n}}^{\tau_{n+1}} \mathbf{E}\left\|b_{x}^{\prime}\left(\tau_{n+1}, \tau_{i}, Y_{i}^{\delta}\right) b\left(\tau_{i}, \tau_{i}, Y_{i}^{\delta}\right)\left(W(s)-W\left(\tau_{n}\right)\right)\right\|_{\mathscr{L}_{2}}^{2} d s<\infty
$$

Из линейного роста $b$ и ограниченности $b_{x}^{\prime}$ получаем, что подынтегральные выражение не превышает $C\left(1+\mathbf{E}\left\|Y_{i}^{\delta}\right\|^{2}\right)$. Поэтому конечность $\mathbf{E}\left\|Y_{i}^{\delta}\right\|^{2}$ можно доказать индукцией по $n$ (остальные слагаемые в правой части (1.5) оцениваются очевидным образом с использованием линейного роста $a$ и $b$ ). 
Заметим, что в данном случае аппроксимации не являются пошаговыми, т.е. для вычисления значения $Y_{n+1}^{\delta}$ в узле $\tau_{n+1}$ надо знать не только предыдущее, $Y_{n}^{\delta}$, но и все остальные до него. Этот эффект обусловлен не специальным выбором схемы аппроксимации, а тем, что, вообще говоря, решения уравнения (1.3) не обладают марковским свойством. Поэтому для уравнений типа Ито-Вольтерра не удается сформулировать утверждение, аналогичное классическому результату Мильштейна о том, что из устойчивости схемы следует ее сходимость, см. [24].

Полагая $Y^{\delta}\left(\tau_{n}\right)=Y_{n}^{\delta}$, мы строим непрерывную интерполяцию

$$
\begin{aligned}
Y^{\delta}(t)= & m^{\delta}(t)+\int_{0}^{t} a\left(t, \tau_{n_{s}}, Y^{\delta}\left(\tau_{n_{s}}\right)\right) d s+\int_{0}^{t} b\left(t, \tau_{n_{s}}, Y^{\delta}\left(\tau_{n_{s}}\right)\right) d W(s) \\
& +\int_{0}^{t} b_{x}^{\prime}\left(t, \tau_{\tau_{n_{s}}}, Y^{\delta}\left(\tau_{n_{s}}\right)\right) \\
& \quad \times b\left(\tau_{n_{s}}, \tau_{n_{s}}, Y^{\delta}\left(\tau_{n_{s}}\right)\right)\left(W(s)-W\left(\tau_{n_{s}}\right)\right) d W(s)
\end{aligned}
$$

где $n_{s}=\max \left\{n: \tau_{n}<s\right\}$. Перечислим предположения на коэффициенты $a, b$ и процесс $m(t)$, которые нам понадобятся при доказательстве сходимости аппроксимаций.

1) Процесс $m(t)$ имеет стохастический дифференциал

$$
d m(t)=\alpha(t) d t+\beta(t) d W(t),
$$

коэффициенты $\alpha(t), \beta(t)$ являются $\mathscr{F}_{t}$-согласованными непрерывными квадратично интегрируемыми процессами в $X$ и $\mathscr{L}_{2}$ соответственно и

$$
\int_{0}^{T} \mathbf{E}\|\beta(t)\|_{\mathscr{L}_{2}}^{4} d t<\infty .
$$

2) Выполнены условия (1.4a) и функция $b$ ограничена.

3) Функции $a, b$ липшицевы по $s$ :

$$
\begin{aligned}
& \|a(t, s, x)-a(t, u, x)\|+\|b(t, s, x)-b(t, u, x)\|_{\mathscr{L}_{2}} \\
& \quad \leqslant C(1+\|x\|)|s-u| .
\end{aligned}
$$

4) Производные $a_{t}^{\prime}, b_{t}^{\prime}$ удовлетворяют условию линейного роста:

$$
\left\|a_{t}^{\prime}(t, s, x)\right\|+\left\|b_{t}^{\prime}(t, s, x)\right\|_{\mathscr{L}_{2}} \leqslant C(1+\|x\|) .
$$

5) Производные $b_{x}^{\prime}, a_{x}^{\prime}$ ограничены, производная $b_{x}^{\prime}$ липшицева по $s$ :

$$
\begin{gathered}
\left\|a_{x}^{\prime}(t, s, x)\right\|_{\mathscr{L}}+\left\|b_{x}^{\prime}(t, s, x)\right\|_{\mathscr{L}\left(X, \mathscr{L}_{2}\right)} \leqslant C, \\
\left\|b_{x}^{\prime}(t, s, x)-b_{x}^{\prime}(t, u, x)\right\|_{\mathscr{L}\left(X, \mathscr{L}_{2}\right)} \leqslant C|s-u| .
\end{gathered}
$$

5) Вторые производные $a_{x x}^{\prime \prime}, b_{x x}^{\prime \prime}$ и функция $b$ ограничены:

$$
\left\|a_{x x}^{\prime \prime}(t, s, x)\right\|_{\mathscr{L}(X \oplus X, X)}+\left\|b_{x x}^{\prime \prime}(t, s, x)\right\|_{\mathscr{L}\left(X \oplus X, \mathscr{L}_{2}\right)}+\|b(t, s, x)\|_{\mathscr{L}_{2}} \leqslant C .
$$


Теорема 1.1. Если коэффиииенть уравнения (1.3) удовлетворяют указанным выше условиям, а также

$$
\mathbf{E}\left\|m^{\delta}(t)-m(t)\right\|^{2} \leqslant C \delta^{2},
$$

то аппроксимачии (1.6) сходятся к решению уравнения (1.3), причем

$$
\mathbf{E}\left\|X(t)-Y^{\delta}(t)\right\|^{2} \leqslant K \delta^{2} .
$$

Д о к аз а т ел ь с т в о. Положим $Z(t)=\mathbf{E}\left\|X(t)-Y^{\delta}(t)\right\|^{2}$. Имеем $Z(t) \leqslant 3\left(\left\|m^{\delta}(t)-m(t)\right\|^{2}+A+B\right)$, где

$$
\begin{aligned}
& A=\mathbf{E}\left\|\int_{0}^{t}\left(a(t, s, X(s))-a\left(t, \tau_{n_{s}}, Y^{\delta}\left(\tau_{n_{s}}\right)\right)\right) d s\right\|^{2}, \\
& B=\mathbf{E}\left\|\int_{0}^{s}\left(b(t, s, X(s))-b\left(t, \tau_{n_{s}}, Y^{\delta}\left(\tau_{n_{s}}\right)\right)\right) d W(s)\right\|^{2} .
\end{aligned}
$$

Схема оценки обоих выражений в основном аналогична: мы разбиваем интегралы на несколько слагаемых так, чтобы в каждом интеграле стояло приращение функции только по одному аргументу; слагаемые затем оцениваются по отдельности. Таким образом,

$$
\begin{aligned}
A & \leqslant C\left(A_{1}+A_{2}+A_{3}\right), \\
A_{1} & =\mathbf{E}\left\|\int_{0}^{t}\left(a\left(t, \tau_{n_{s}}, X\left(\tau_{n_{s}}\right)\right)-a\left(t, \tau_{n_{s}}, Y^{\delta}\left(\tau_{n_{s}}\right)\right)\right) d s\right\|^{2} \\
& \leqslant C \int_{0}^{t} \mathbf{E}\left\|a\left(t, \tau_{n_{s}}, X\left(\tau_{n_{s}}\right)\right)-a\left(t, \tau_{n_{s}}, Y^{\delta}\left(\tau_{n_{s}}\right)\right)\right\|^{2} d s \leqslant C \int_{0}^{t} Z\left(\tau_{n_{s}}\right) d s, \\
A_{2} & =\mathbf{E}\left\|\int_{0}^{t}\left(a\left(t, s, X\left(\tau_{n_{s}}\right)\right)-a\left(t, \tau_{n_{s}}, X\left(\tau_{n_{s}}\right)\right)\right) d s\right\|^{2} \\
& \leqslant C \int_{0}^{t} \mathbf{E}\left\|a\left(t, s, X\left(\tau_{n_{s}}\right)\right)-a\left(t, \tau_{n_{s}}, X\left(\tau_{n_{s}}\right)\right)\right\|^{2} d s \\
& \left.\leqslant C \int_{0}^{t} \mathbf{E}\left(1+\| X\left(\tau_{n_{s}}\right)\right) \|^{2}\right) \delta^{2} d s \leqslant C \delta^{2}, \\
A_{3} & =\mathbf{E}\left\|\int_{0}^{t}\left(a\left(t, s, X\left(\tau_{n_{s}}\right)\right)-a(t, s, X(s))\right) d s\right\|^{2} .
\end{aligned}
$$

Чтобы оценить $A_{3}$, можно воспользоваться формулой Ито (см. [5]). Действительно, процесс $X(t)$ равняется сумме непрерывного процесса с ограниченной вариацией и квадратично интегрируемого мартингала, поэтому

$$
\begin{gathered}
a(t, s, X(s))-a\left(t, s, X\left(\tau_{n_{s}}\right)\right) \\
=\int_{\tau_{n_{s}}}^{s} a_{x}^{\prime}(t, s, X(u))\left(\alpha(u)+a(u, u, X(u))+\int_{0}^{u} a_{t}^{\prime}(u, v, X(v)) d v\right. \\
\left.\quad+\int_{0}^{u} b_{t}^{\prime}(u, v, X(v)) d W(v)\right) d u
\end{gathered}
$$




$$
\begin{aligned}
& +\int_{\tau_{n_{s}}}^{s} a_{x}^{\prime}(t, s, X(u))(\beta(u)+b(u, u, X(u))) d W(u) \\
& +\int_{\tau_{n_{s}}}^{s} a_{x x}^{\prime \prime}(t, s, X(u))\left((\beta(u)+b(u, u, X(u)))(\beta(u)+b(u, u, X(u)))^{\top}\right) d u .
\end{aligned}
$$

Здесь для $a \in \mathscr{L}(X \oplus X, X), b \in \mathscr{L}_{2}$ мы упростили запись $\left(\left\{e_{k}, k \geqslant 1\right\}-\right.$ ортонормированный базис в $X)$ :

$$
a\left(b b^{\top}\right):=\sum_{k=1}^{\infty} a\left(b e_{k}, b e_{k}\right) .
$$

Последнее означает не скалярное произведение, а аргументы билинейной формы. Оценим это выражение:

$\left\|a\left(b b^{\top}\right)\right\| \leqslant \sum_{k=1}^{\infty}\left\|a\left(b e_{k}, b e_{k}\right)\right\| \leqslant\|a\|_{\mathscr{L}(X \oplus X, X)} \sum_{k=1}^{\infty}\left\|b e_{k}\right\|^{2} \leqslant\|a\|_{\mathscr{L}(X \oplus X, X)}\|b\|_{\mathscr{L}_{2}}$.

Теперь разобьем $A_{3}$ на слагаемые, отвечающие слагаемым в формуле Ито, и каждое оценим по отдельности:

$$
\begin{aligned}
& A_{31}= \mathbf{E}\left\|\int_{0}^{t} \int_{\tau_{n_{s}}}^{s} a_{x}^{\prime}(t, s, X(u))(\alpha(u)+a(u, u, X(u))) d u d s\right\|^{2} \\
& \leqslant C \mathbf{E} \int_{0}^{t}\left(s-\tau_{n_{s}}\right) \int_{\tau_{n_{s}}}^{s}\left\|a_{x}^{\prime}(t, s, X(u))\right\|_{\mathscr{L}}^{2} \\
& \times\left(\|\alpha(u)\|^{2}+\|a(u, u, X(u))\|\right)^{2} d u d s \\
& \leqslant C \delta \int_{0}^{t} \int_{\tau_{n_{s}}}^{s} \mathbf{E}\left(1+\|X(u)\|^{2}\right) d u d s \leqslant C \delta^{2}, \\
& A_{32}=\mathbf{E}\left\|\int_{0}^{t} \int_{\tau_{n_{s}}}^{s} a_{x}^{\prime}(t, s, X(u))(\beta(u)+b(u, u, X(u)) d W(u)) d s\right\|^{2} \\
&=\mathbf{E}\left\|\int_{0}^{t} \int_{u}^{\tau_{n_{u}}+\delta} a_{x}^{\prime}(t, s, X(u))(\beta(u)+b(u, u, X(u))) d s d W(u)\right\|^{2} \\
& \leqslant C \delta \int_{0}^{t} \int_{u}^{\tau_{n_{u}}+\delta} \mathbf{E}\left\|a_{x}^{\prime}(t, s, X(u))\right\|_{\mathscr{L}}^{2} \\
& A_{33}=\mathbf{E} \| \int_{0}^{t} \int_{\tau_{n_{s}}}^{s} a_{x x}^{\prime \prime}(t, s, X(u)) \\
&\left.\times C \int_{\tau_{n_{s}}}^{s}\left\|a_{x x}^{\prime \prime}\right\|_{\mathscr{L}(X \oplus X, X)} \mathbf{E}\left(\|b(u, u, X(u))\|_{\mathscr{L}_{2}}^{4}+\|\beta(u)\|_{\mathscr{L}_{2}}^{4}\right) d u \leqslant C \delta^{2},\|b(u, u, X(u))\|_{\mathscr{L}_{2}}^{2}\right) d s d u \leqslant C \delta^{2}, \\
& A_{34}=\mathbf{E}\left\|\int_{0}^{t} \int_{\tau_{n_{s}}}^{s} a_{x}^{\prime}(t, s, X(u)) \int_{0}^{u} a_{t}^{\prime}(s, v, X(v)) d v d u d s\right\|^{2}
\end{aligned}
$$




$$
\begin{aligned}
& \leqslant C \delta \int_{0}^{t} \int_{\tau_{n_{s}}}^{s} \int_{0}^{u} \mathbf{E}\left\|a_{x}^{\prime}(t, s, X(s))\right\|_{\mathscr{L}}^{2}\left\|a_{t}^{\prime}(s, v, X(v))\right\|^{2} d v d u d s \\
& \leqslant C \delta \int_{0}^{t} \int_{\tau_{n_{s}}}^{s} \int_{0}^{u} \mathbf{E}\left(1+\|X(v)\|^{2}\right) d v d u d s \leqslant C \delta^{2}, \\
A_{35} & =\mathbf{E}\left\|\int_{0}^{t} \int_{\tau_{n_{s}}}^{s} a_{x}^{\prime}(t, s, X(u)) \int_{0}^{u} b_{t}^{\prime}(s, v, X(v)) d W(v) d u d s\right\|^{2} \\
& \leqslant C \delta \int_{0}^{t} \int_{\tau_{n_{s}}}^{s} \int_{0}^{u} \mathbf{E}\left\|a_{x}^{\prime}(t, s, X(s))\right\|_{\mathscr{L}}^{2}\left\|b_{t}^{\prime}(s, v, X(v))\right\|_{\mathscr{L}_{2}}^{2} d v d u d s \\
& \leqslant C \delta \int_{0}^{t} \int_{\tau_{n_{s}}}^{s} \int_{0}^{u} \mathbf{E}\left(1+\|X(v)\|^{2}\right) d v d u d s \leqslant C \delta^{2} .
\end{aligned}
$$

Полностью аналогично, за исключением того, что вместо оценки

$$
\mathbf{E}\left\|\int_{0}^{t} \cdots d s\right\|^{2} \leqslant t \int_{0}^{t} \mathbf{E}\|\cdots\|^{2} d s
$$

мы теперь используем изометрическое свойство

$$
\mathbf{E}\left\|\int_{0}^{t} \cdots d W(s)\right\|^{2}=\int_{0}^{t} \mathbf{E}\|\cdots\|_{\mathscr{L}_{2}}^{2} d s
$$

оценивается слагаемое $B$ :

$$
\begin{aligned}
B & \leqslant C\left(B_{1}+B_{2}+B_{3}+B_{4}\right) \\
B_{1} & =\mathbf{E}\left\|\int_{0}^{t}\left(b\left(t, \tau_{n_{s}}, X\left(\tau_{n_{s}}\right)\right)-b\left(t, \tau_{n_{s}}, Y^{\delta}\left(\tau_{n_{s}}\right)\right)\right) d s\right\|^{2} \\
& \leqslant C \int_{0}^{t} \mathbf{E}\left\|b\left(t, \tau_{n_{s}}, X\left(\tau_{n_{s}}\right)\right)-b\left(t, \tau_{n_{s}}, Y^{\delta}\left(\tau_{n_{s}}\right)\right)\right\|^{2} d s \leqslant C \int_{0}^{t} Z\left(\tau_{n_{s}}\right) d s \\
B_{2} & =\mathbf{E}\left\|\int_{0}^{t}\left(b\left(t, s, X\left(\tau_{n_{s}}\right)\right)-b\left(t, \tau_{n_{s}}, X\left(\tau_{n_{s}}\right)\right)\right) d s\right\|^{2} \\
& \leqslant C \int_{0}^{t} \mathbf{E}\left\|b\left(t, s, X\left(\tau_{n_{s}}\right)\right)-b\left(t, \tau_{n_{s}}, X\left(\tau_{n_{s}}\right)\right)\right\|^{2} d s \\
& \leqslant C \int_{0}^{t} \mathbf{E}\left(1+\left\|X\left(\tau_{n_{s}}\right)\right\|^{2}\right) \delta^{2} d s \leqslant C \delta^{2}, \\
B_{3} & =\mathbf{E} \| \int_{0}^{t}\left(b(t, s, X(s))-b\left(t, s, X\left(\tau_{n_{s}}\right)\right)\right) d W(s) \\
B_{4} & =\mathbf{E} \| \int_{0}^{t} \int_{\tau_{n_{s}}}^{s}\left(b_{x}^{\prime}(t, s, X(u)) b(u, u, X(u))\right. \\
& -\int_{\tau_{n_{s}}}^{s} b_{x}^{\prime}(t, s, X(u)) b(u, u, X(u)) d W(u) d W(s) \|^{2},
\end{aligned}
$$

Слагаемое $B_{3}$ оцениваются по аналогии с оценками для $A_{3}$ (заметим, что одно слагаемое в формуле Ито, содержащее повторный интеграл по винеровскому процессу, при этом сокращается; именно это слагаемое 
имеет более низкий порядок сходимости к нулю). Заметим, что функция $b_{x}^{\prime}(t, s, x) b(s, s, x)$ при сделанных предположениях будет липшицевой и линейного роста, поэтому слагаемое $B_{4}$ можно оценить таким же методом, каким оценивались $A, B$. Таким образом, получаем оценку

$$
Z(t) \leqslant C\left(\delta^{2}+\int_{0}^{t} Z\left(\tau_{n_{s}}\right) d s\right)
$$

откуда в силу леммы Гронуолла следует $Z(t) \leqslant C \delta^{2}$ с константой, не зависящей от $\delta$. Теорема 1.1 доказана.

1.2. Аппроксимации полулинейных уравнений эволюционного типа по схеме Мильштейна. Вернемся к уравнению (1.1). Как уже было сказано, его сильное решение является мягким, т.е. удовлетворяет уравнению

$$
X(t)=U(t) X_{0}+\int_{0}^{t} U(t-s) a(s, X(s)) d s+\int_{0}^{t} U(t-s) b(s, X(s)) d W(s) .
$$

Мы наложим следующие условия, гарантирующие существование, единственность и непрерывность решения уравнений (1.1), (1.9) (доказательство можно найти в [5]).

(A) Выполнены условия липшицевости и линейного роста:

$$
\begin{gathered}
\|a(t, x)\|_{X}+\|b(t, x)\|_{\mathscr{L}_{2}} \leqslant C\left(1+\|x\|_{X}\right), \\
\|a(t, x)-a(t, y)\|_{X}+\|b(t, x)-b(t, y)\|_{\mathscr{L}_{2}} \leqslant C\|x-y\|_{X},
\end{gathered}
$$

(B) Оператор $A$ порождает сильно непрерывную полугруппу операторов $\{U(t), 0 \leqslant t \leqslant T\}$ на $X$.

(C) Выполнены условия «гладкости» коэффициентов $a, b$ :

$$
\|A a(t, x)\|+\|A b(t, x)\|_{\mathscr{L}_{2}} \leqslant C(1+\|x\|) .
$$

3 а м е ч а н и е 1.3. Для аппроксимаций уравнения (1.9) по схеме Эйлера последнее условие избыточно. Можно потребовать, чтобы коэффициенты $a, b$ «сглаживали» не все $x$, а только «гладкие» $x$, т.е.

$$
\|A a(t, x)\|+\|A b(t, x)\|_{\mathscr{L}_{2}} \leqslant C(1+\|A x\|)
$$

для $x \in D(A)$. K сожалению, эти условия не гарантируют существования стохастического дифференциала у решения (1.9) и поэтому не подходят для схемы Мильштейна, которая основана на формуле Ито.

В предположении существования и ограниченности производной $b_{x}^{\prime}$ аппроксимации по схеме Мильштейна для уравнения (1.9) можно записать, основываясь на схеме для уравнений Ито-Вольтерра:

$$
\begin{aligned}
Y_{n+1}^{\delta}= & U\left(\tau_{n+1}\right) Y_{0}^{\delta} \\
& \quad+\sum_{i=0}^{n} U\left(\tau_{n+1}-\tau_{i}\right)\left(a\left(\tau_{i}, Y_{i}^{\delta}\right) \delta+b\left(\tau_{i}, Y_{i}^{\delta}\right)\left(W\left(\tau_{i+1}\right)-W\left(\tau_{i}\right)\right)\right. \\
& \left.\quad+\int_{\tau_{i}}^{\tau_{i+1}} b_{x}^{\prime}\left(\tau_{i}, Y_{i}^{\delta}\right) b\left(\tau_{i}, Y_{i}^{\delta}\right)\left(W(s)-W\left(\tau_{i}\right)\right) d W(s)\right) .
\end{aligned}
$$


Используя полугрупповое свойство, последнее можно переписать так:

$$
\begin{aligned}
Y_{n+1}^{\delta}=U(\delta)( & Y_{n}^{\delta}+a\left(\tau_{n}, Y_{n}^{\delta}\right) \delta+b\left(\tau_{n}, Y_{n}^{\delta}\right)\left(W\left(\tau_{n+1}\right)-W\left(\tau_{n}\right)\right) \\
& +\int_{\tau_{n}}^{\tau_{n+1}} b_{x}^{\prime}\left(\tau_{n}, Y_{n}^{\delta}\right) b\left(\tau_{n}, Y_{n}^{\delta}\right) \\
& \left.\quad \times\left(W(s)-W\left(\tau_{n}\right)\right) d W(s)\right), \quad n \geqslant 0 .
\end{aligned}
$$

Заметим, что аппроксимации в данном случае являются пошаговыми, благодаря марковскому свойству решений уравнения (1.1).

Сделаем непрерывную интерполяцию аппроксимаций (1.12):

$$
\begin{aligned}
Y^{\delta}(t)=U(t) Y_{0}^{\delta}+\int_{0}^{t} U\left(t-\tau_{n_{s}}\right) & \left(a\left(\tau_{n_{s}}, Y^{\delta}\left(\tau_{n_{s}}\right)\right) d s+b\left(\tau_{n_{s}}, Y^{\delta}\left(\tau_{n_{s}}\right)\right) d W(s)\right. \\
& +b_{x}^{\prime}\left(\tau_{n_{s}}, Y^{\delta}\left(\tau_{n_{s}}\right)\right) b\left(\tau_{n_{s}}, Y^{\delta}\left(\tau_{n_{s}}\right)\right) \\
& \left.\times\left(W(s)-W\left(\tau_{n_{s}}\right)\right) d W(s)\right) .
\end{aligned}
$$

Предположим, что коэффициенты уравнения (1.1) удовлетворяют условиям $(\mathrm{A})-(\mathrm{C})$ и следующим условиям сходимости схемы Мильштейна для обычных СДУ.

1) Функции $a, b$ липшицевы по $t$ :

$$
\|a(t, x)-a(s, x)\|+\|b(t, x)-b(s, x)\|_{\mathscr{L}_{2}} \leqslant C|t-s|(1+\|x\|) .
$$

2) Производные $b_{x}^{\prime}, a_{x}^{\prime}$ ограничены, производная $b_{x}^{\prime}$ липшицева по $t$ :

$$
\begin{array}{r}
\left\|a_{x}^{\prime}(t, x)\right\|_{\mathscr{L}}+\left\|b_{x}^{\prime}(t, x)\right\|_{\mathscr{L}\left(X, \mathscr{L}_{2}\right)} \leqslant C, \\
\left\|b_{x}^{\prime}(t, x)-b_{x}^{\prime}(s, x)\right\|_{\mathscr{L}\left(X, \mathscr{L}_{2}\right)} \leqslant C|t-s| .
\end{array}
$$

3) Вторые производные $a_{x x}^{\prime \prime}, b_{x x}^{\prime \prime}$ и функция $b$ ограничены:

$$
\left\|a_{x x}^{\prime \prime}(t, x)\right\|_{\mathscr{L}(X \oplus X, X)}+\left\|b_{x x}^{\prime \prime}(t, x)\right\|_{\mathscr{L}\left(X \oplus X, \mathscr{L}_{2}\right)}+\|b(t, x)\|_{\mathscr{L}_{2}} \leqslant C .
$$

Предположим также, что $\mathbf{E}\left\|A X_{0}\right\|^{2}<\infty$.

Теорема 1.2. Аппроксимачии (1.13) сходятся к решению уравнения (1.1), причем

$$
\mathbf{E}\left\|X(t)-Y^{\delta}(t)\right\|^{2} \leqslant K \delta^{2} .
$$

Д о к а з а т е л ь с т в о. В книге [5] доказано, что при выполнении условий $(\mathrm{A})-(\mathrm{C})$ существует сильное потраекторно единственное решение уравнения $X(t)$, которое принадлежит $D(A)$ почти наверное почти для всех $t$. При этом $X(t)$ имеет стохастический дифференциал

$$
d X(t)=\mathbb{I}_{X(t) \in D(A)} A X(t) d t+a(t, X(t)) d t+b(t, X(t)) d W(t),
$$


используемый далее в формуле Ито, и при $\mathbf{E}\left\|A X_{0}\right\|^{2}<\infty$ справедливо

$$
\sup _{t \in[0, T]} \mathbf{E}\|A X(t)\|^{2}<\infty
$$

Имеем

$$
\mathbf{E}\left\|X(t)-Y^{\delta}(t)\right\|^{2} \leqslant 2\left(\mathbf{E}\left\|X(t)-X_{1}(t)\right\|^{2}+\mathbf{E}\left\|X_{1}(t)-Y^{\delta}(t)\right\|^{2}\right),
$$

где

$$
X_{1}(t)=U(t) X_{0}+\int_{0}^{t} U\left(t-\tau_{n_{s}}\right)(a(s, X(s)) d s+b(s, X(s)) d W(s)) .
$$

Разность $\mathbf{E}\left\|X_{1}(t)-Y^{\delta}(t)\right\|^{2}$ оценивается точно так же, как в теореме 1.1, за исключением того, что при применении формулы Ито возникают иные слагаемые, которые мы оценим:

$$
\begin{aligned}
& \mathbf{E}\left\|\int_{0}^{t} U\left(t-\tau_{n_{s}}\right) \int_{\tau_{n_{s}}}^{s} a_{x}^{\prime}(s, X(u)) A X(u) d u d s\right\|^{2} \\
& \quad \leqslant C \delta \int_{0}^{t} \int_{\tau_{n_{s}}}^{s}\left\|U\left(t-\tau_{n_{s}}\right)\right\|^{2} \mathbf{E}\left\|a_{x}^{\prime}(s, X(u))\right\|^{2}\|A X(u)\|^{2} d u d s \leqslant C \delta^{2}, \\
& \mathbf{E}\left\|\int_{0}^{t} U\left(t-\tau_{n_{s}}\right) \int_{\tau_{n_{s}}}^{s} b_{x}^{\prime}(s, X(u)) A X(u) d u d W(s)\right\|^{2} \\
& \quad \leqslant \delta \int_{0}^{t} \int_{\tau_{n_{s}}}^{s}\left\|U\left(t-\tau_{n_{s}}\right)\right\|^{2} \mathbf{E}\left\|b_{x}^{\prime}(s, X(u))\right\|_{\mathscr{L}\left(X, \mathscr{L}_{2}\right)}^{2}\|A X(u)\|^{2} d u d s \leqslant C \delta^{2} .
\end{aligned}
$$

Далее,

$$
\begin{aligned}
& \mathbf{E}\left\|X(t)-X_{1}(t)\right\|^{2} \\
& =\mathbf{E}\left\|\int_{0}^{t}\left(U(t-s)-U\left(t-\tau_{n_{s}}\right)\right)(a(s, X(s)) d s+b(s, X(s)) d W(s))\right\|^{2} \\
& =\mathbf{E}\left\|\int_{0}^{t}\left(\int_{\tau_{n_{s}}}^{s} U(t-v) A d v\right)(a(s, X(s)) d s+b(s, X(s)) d W(s))\right\|^{2} \\
& \leqslant C\left(\delta \int_{0}^{t} \int_{\tau_{n_{s}}}^{s}\|U(t-v)\|\right. \\
& \left.\quad \times\left(\mathbf{E}\|A a(s, X(s))\|^{2}+\mathbf{E}\|A b(s, X(s))\|_{\mathscr{L}_{2}}^{2}\right) d v d s\right) \\
& \leqslant C \delta \int_{0}^{t} \int_{\tau_{n_{s}}}^{s} \mathbf{E}\left(1+\|X(s)\|^{2}\right) d v d s \leqslant C \delta^{2} .
\end{aligned}
$$

Теорема 1.2 доказана.

3 а м е ч а н и е 1.4. Нетрудно видеть, что предложенный метод приближенного решения уравнения (1.1) является не чем иным, как 
известным (по крайней мере, для неслучайных уравнений) методом «splitting-up». Сначала уравнение (1.1) разбивается на два:

$$
d X^{1}(t)=a\left(t, X^{1}(t)\right) d t+b\left(t, X^{1}(t)\right) d W(t), \quad d X^{2}(t)=A X^{2}(t) d t .
$$

Потом последовательно на каждом из отрезков разбиения сначала приближенно решается первое уравнение:

$$
\begin{aligned}
Y_{n+1}^{\delta, 1}= & Y_{n}^{\delta}+a\left(\tau_{n}, Y_{n}^{\delta}\right) \delta+b\left(\tau_{n}, Y_{n}^{\delta}\right)\left(W\left(\tau_{n+1}\right)-W\left(\tau_{n}\right)\right) \\
& +\int_{\tau_{n}}^{\tau_{n+1}} b_{x}^{\prime}\left(\tau_{n}, Y_{n}^{\delta}\right) b\left(\tau_{n}, Y_{n}^{\delta}\right)\left(W(s)-W\left(\tau_{n}\right)\right) d W(s),
\end{aligned}
$$

потом результат подставляется во второе:

$$
Y_{n+1}^{\delta, 2}=U(\delta) Y_{n+1}^{\delta, 1}
$$

и это дает приближенное решение уравнения (1.1) (формула для $Y_{n+1}^{\delta, 2}$ совпадает с (1.12)).

3 а м е ч а н и е 1.5. Еще одно важное замечание состоит в том, что нам не надо уметь решать второе уравнение точно, а только приближенно. Более точно, в формуле (1.12) можно заменить оператор $U(\delta)$ оператором $\widetilde{U}_{\delta}$ таким, что

$$
\left\|U(\delta)-\tilde{U}_{\delta}\right\|_{\mathscr{L}} \leqslant C \delta^{2}
$$

с константой, не зависящей от $\delta$. Действительно, из этой оценки легко следует

$$
\left\|U^{n}(\delta)-\tilde{U}_{\delta}^{n}\right\|_{\mathscr{L}} \leqslant C \delta
$$

Обозначим модифицированные аппроксимации $\tilde{Y}_{n}^{\delta}$ и запишем

$$
\begin{aligned}
\tilde{Y}_{n+1}^{\delta}=\tilde{U}_{\delta}^{n} Y_{0}^{\delta}+\sum_{i=0}^{n} \tilde{U}_{\delta}^{n+1-i}\left(a\left(\tau_{i}, Y_{i}^{\delta}\right) \delta+b\left(\tau_{i}, Y_{i}^{\delta}\right)\left(W\left(\tau_{i+1}\right)-W\left(\tau_{i}\right)\right)\right. \\
\left.+\int_{\tau_{i}}^{\tau_{i+1}} b_{x}^{\prime}\left(\tau_{i}, Y_{i}^{\delta}\right) b\left(\tau_{i}, Y_{i}^{\delta}\right)\left(W(s)-W\left(\tau_{i}\right)\right) d W(s)\right) .
\end{aligned}
$$

Сравнивая это с формулой (1.11), получим

$$
\mathbf{E}\left\|Y_{n+1}^{\delta}-\tilde{Y}_{n+1}^{\delta}\right\|^{2} \leqslant C\left(\delta^{2}+\delta \sum_{i=1}^{n} \mathbf{E}\left\|Y_{i}^{\delta}-\tilde{Y}_{i}^{\delta}\right\|^{2}\right)
$$

и, используя дискретный вариант леммы Гронуолла, приходим к неравенству

$$
\mathbf{E}\left\|Y_{n}^{\delta}-\tilde{Y}_{n}^{\delta}\right\|^{2} \leqslant C \delta^{2} .
$$




\section{2. Аппроксимации конечномерными процессами}

Предложенные в предыдущем разделе схемы аппроксимации решений стохастических дифференциальных уравнений являются прямым аналогом соответствующих схем в конечномерном случае. Тем не менее, в бесконечномерном случае априори не ясно, не потребует ли отыскание приближенного решения бесконечного числа операций. Поэтому естественным образом возникает вопрос об аппроксимации решений стохастического эволюционного уравнения (1.1) конечномерными процессами (решениями конечномерных уравнений).

Как и в предыдущем разделе, начнем с рассмотрения уравнения Ито-Вольтерра

$$
X(t)=m(t)+\int_{0}^{t} a(t, s, X(s)) d s+\int_{0}^{t} b(t, s, X(s)) d W(s) .
$$

Предположим, что его коэффициенты непрерывны по совокупности переменных и удовлетворяют условиям липшицевости и линейного роста:

$$
\begin{gathered}
\|a(t, s, x)\|+\|b(t, s, x)\|_{\mathscr{L}_{2}} \leqslant C(1+\|x\|), \\
\|a(t, s, x)-a(t, s, y)\|+\|b(t, s, x)-b(t, s, y)\|_{\mathscr{L}_{2}} \leqslant C\|x-y\|,
\end{gathered}
$$

и существует возрастающая функция $h(t), t>0$, такая, что $h(t) \rightarrow 0$ при $t \rightarrow 0$ и

$$
\|a(t, u, x)-a(s, u, x)\|+\|b(t, u, x)-b(s, u, x)\| \leqslant h(t-s)(1+\|x\|) .
$$

Процесс $m(t)$ будем считать согласованным и непрерывным в среднем квадратическом.

Пусть $\left\{e_{n}, n \geqslant 1\right\}$ - ортонормированный базис в $X$, обозначим $E_{n}=$ $\operatorname{span}\left\{e_{i}, i \leqslant n\right\}, P_{n}$ - ортогональный проектор на $E_{n}$. Конечномерные аппроксимации решения уравнения (2.1) построим следующим образом:

$$
X_{n}(t)=P_{n} m(t)+\int_{0}^{t} P_{n} a\left(t, s, X_{n}(s)\right) d s+\int_{0}^{t} P_{n} b\left(t, s, X_{n}(s)\right) P_{n} d W(s) .
$$

Вначале докажем сходимость $\mathbf{E}\left\|X_{n}(t)-X(t)\right\|^{2} \rightarrow 0, n \rightarrow \infty$, в более общем случае.

Пусть $X_{n}$ - решение уравнения

$$
X_{n}(t)=m_{n}(t)+\int_{0}^{t} a_{n}\left(t, s, X_{n}(s)\right) d s+\int_{0}^{t} b_{n}\left(t, s, X_{n}(s)\right) d W(s),
$$

в котором $m_{n}$ - непрерывный в среднем квадратическом согласованный процесс и коэффициенты которого удовлетворяют условиям

$$
\begin{gathered}
\left\|a_{n}(t, s, x)\right\|+\left\|b_{n}(t, s, x)\right\|_{\mathscr{L}_{2}} \leqslant C(1+\|x\|), \\
\left\|a_{n}(t, s, x)-a_{n}(t, s, y)\right\|+\left\|b_{n}(t, s, x)-b_{n}(t, s, y)\right\|_{\mathscr{L}_{2}} \leqslant C\|x-y\|
\end{gathered}
$$


с константой, не зависящей от $n$; кроме того, пусть существует такая возрастающая функция $h(t), t>0$, что $h(t) \rightarrow 0$ при $t \rightarrow 0$ и

$$
\begin{aligned}
& \left\|a_{n}(t, u, x)-a_{n}(s, u, x)\right\|+\left\|b_{n}(t, u, x)-b_{n}(s, u, x)\right\| \\
& \quad \leqslant h(t-s)(1+\|x\|)
\end{aligned}
$$

$$
\mathbf{E}\left\|m_{n}(t)-m_{n}(s)\right\|^{2} \leqslant h^{2}(t-s) .
$$

Далее, предположим, что для всех $t, s \in[0, T]$ и $x \in X$

$$
\begin{gathered}
\left\|a(t, s, x)-a_{n}(t, s, x)\right\|+\left\|b(t, s, x)-b_{n}(t, s, x)\right\|_{\mathscr{L}_{2}} \longrightarrow 0, \quad n \rightarrow \infty \\
\mathbf{E}\left\|m(t)-m_{n}(t)\right\|^{2} \longrightarrow 0, \quad n \rightarrow \infty
\end{gathered}
$$

Теорема 2.1. При предположениях (2.2), (2.4), (2.5) справедлива равномерная на отрезке $[0, T]$ сходимость

$$
\mathbf{E}\left\|X(t)-X_{n}(t)\right\|^{2} \longrightarrow 0, \quad n \rightarrow \infty .
$$

Д ок а з тельст в о. Как и ранее, легко показать, что $\mathbf{E}\|X(t)\|^{2}, \mathbf{E}\left\|X_{n}(t)\right\|^{2}$ ограничены по $n$ и $t$. Без ограничения общности будем предполагать, что

$$
\mathbf{E}\|m(t)-m(s)\|^{2} \leqslant h^{2}(t-s) .
$$

Отсюда непосредственно следует, что

$$
\mathbf{E}\left\|X_{n}(t)-X_{n}(s)\right\|^{2} \leqslant C h_{0}(t-s), \quad \mathbf{E}\|X(t)-X(s)\|^{2} \leqslant C h_{0}(t-s),
$$

где $h_{0}(t)=\max \left\{h^{2}(t), t\right\}$. Для натурального $N$ положим $\delta=T / N$, зададимся разбиением $\tau_{k}=k \delta$ отрезка $[0, T]$ и построим процессы

$$
\begin{aligned}
& X^{\delta}(t)=m(t)+\int_{0}^{t} a\left(t, s, X^{\delta}\left(\tau_{n_{s}}\right)\right) d s+\int_{0}^{t} b\left(t, s, X^{\delta}\left(\tau_{n_{s}}\right)\right) d W(s), \\
& X_{n}^{\delta}(t)=m_{n}(t)+\int_{0}^{t} a_{n}\left(t, s, X_{n}^{\delta}\left(\tau_{n_{s}}\right)\right) d s+\int_{0}^{t} b_{n}\left(t, s, X_{n}^{\delta}\left(\tau_{n_{s}}\right)\right) d W(s),
\end{aligned}
$$

где, как и прежде, $n_{s}=\max \left\{n: \tau_{n}<s\right\}$. Имеем

$$
\begin{gathered}
Z_{n}^{\delta}(t):=\mathbf{E}\left\|X_{n}^{\delta}(t)-X_{n}(t)\right\|^{2} \leqslant C\left(A_{1}+A_{2}\right), \\
A_{1}=\mathbf{E} \int_{0}^{t}\left(\left\|a_{n}\left(t, s, X_{n}\left(\tau_{n_{s}}\right)\right)-a_{n}\left(t, s, X_{n}(s)\right)\right\|^{2}\right. \\
\left.+\left\|b_{n}\left(t, s, X_{n}\left(\tau_{n_{s}}\right)\right)-b_{n}\left(t, s, X_{n}(s)\right)\right\|_{\mathscr{L}_{2}}^{2}\right) d s \\
\leqslant C \int_{0}^{t} \mathbf{E}\left\|X_{n}\left(\tau_{n_{s}}\right)-X_{n}(s)\right\|^{2} d s \leqslant C h_{0}(\delta),
\end{gathered}
$$




$$
\begin{aligned}
A_{2}= & \mathbf{E} \int_{0}^{t}\left(\left\|a_{n}\left(t, s, X_{n}\left(\tau_{n_{s}}\right)\right)-a_{n}\left(t, s, X_{n}^{\delta}\left(\tau_{n_{s}}\right)\right)\right\|^{2}\right. \\
& \left.+\left\|b_{n}\left(t, s, X_{n}\left(\tau_{n_{s}}\right)\right)-b_{n}\left(t, s, X_{n}^{\delta}\left(\tau_{n_{s}}\right)\right)\right\|_{\mathscr{L}_{2}}^{2}\right) d s \\
\leqslant & C \int_{0}^{t} \mathbf{E}\left\|X_{n}\left(\tau_{n_{s}}\right)-X_{n}^{\delta}\left(\tau_{n_{s}}\right)\right\|^{2} d s=C \int_{0}^{t} Z_{n}^{\delta}\left(\tau_{n_{s}}\right) d s .
\end{aligned}
$$

Таким образом,

$$
Z_{n}^{\delta}(t) \leqslant C\left(h_{0}(\delta)+\int_{0}^{t} Z_{n}^{\delta}\left(\tau_{n_{s}}\right) d s\right)
$$

откуда с применением леммы Гронуолла $Z_{n}^{\delta}(t) \leqslant C h_{0}(\delta)$. Заметим, что константа тут зависит лишь от констант липшицевости и линейного роста коэффициентов, поэтому не зависит от $n, \delta$. Аналогично получаем и $\mathbf{E}\left\|X(t)-X^{\delta}(t)\right\|^{2} \leqslant C h_{0}(\delta)$. Далее,

$$
\begin{gathered}
\mathbf{E}\left\|X_{n}^{\delta}\left(\tau_{k+1}\right)-X^{\delta}\left(\tau_{k+1}\right)\right\|^{2} \leqslant C \mathbf{E}\left(\left\|m(t)-m_{n}(t)\right\|^{2}+B_{1}+B_{2}\right), \\
B_{1}=\mathbf{E} \int_{0}^{\tau_{k+1}}\left(\left\|a\left(t, s, X^{\delta}\left(\tau_{n_{s}}\right)\right)-a_{n}\left(t, s, X^{\delta}\left(\tau_{n_{s}}\right)\right)\right\|^{2}\right. \\
\left.\quad+\left\|\left(t, s, X\left(\tau_{n_{s}}\right)\right)-b_{n}\left(t, s, X\left(\tau_{n_{s}}\right)\right)\right\|_{\mathscr{L}_{2}}^{2}\right) d s, \\
B_{2}=\mathbf{E} \int_{0}^{\tau_{k+1}}\left(\left\|a_{n}\left(t, s, X^{\delta}\left(\tau_{n_{s}}\right)\right)-a_{n}\left(t, s, X_{n}^{\delta}\left(\tau_{n_{s}}\right)\right)\right\|^{2}\right. \\
\left.\quad+\left\|b_{n}\left(t, s, X^{\delta}\left(\tau_{n_{s}}\right)\right)-b_{n}\left(t, s, X_{n}^{\delta}\left(\tau_{n_{s}}\right)\right)\right\|_{\mathscr{L}_{2}}^{2}\right) d s \\
\leqslant C \sum_{i=0}^{k} \mathbf{E}\left\|X^{\delta}\left(\tau_{i}\right)-X_{n}^{\delta}\left(\tau_{i}\right)\right\|^{2} .
\end{gathered}
$$

Слагаемое $B_{1}$ стремится к нулю при $n \rightarrow \infty$ по теореме Лебега о мажорируемой сходимости (интегрируемая мажоранта $-C\left(1+\left\|X\left(\tau_{n_{s}}\right)\right\|^{2}\right)$ ). Сходимость $B_{2}$ к нулю при $n \rightarrow \infty$ можно доказать индукцией по $k$. Таким образом, получаем

$$
\limsup _{n \rightarrow \infty} \mathbf{E}\left\|X\left(\tau_{k}\right)-X_{n}\left(\tau_{k}\right)\right\|^{2} \leqslant C h_{0}(\delta) .
$$

Из непрерывности в среднем квадратическом $X(t)$ и $X_{n}(t)$ (оценки $(2.6)$ ) получаем $\lim \sup _{n \rightarrow \infty} \mathbf{E}\left\|X(t)-X_{n}(t)\right\|^{2} \leqslant C h_{0}(\delta)$. Тут левая часть не зависит от $\delta$, а константа в правой части не зависит от $t$, поэтому, переходя к пределу при $\delta \rightarrow 0$, получаем требуемое. Теорема 2.1 доказана.

3 а м е ч а н и е 2.1. Заметим, что в данном случае недостаточно стандартных для этой статьи рассуждений с использованием леммы Гронуолла. Дело в том, что, используя лемму Гронуолла, мы стремимся 
получить оценку вроде $\mathbf{E}\left\|X_{n}(t)-X(t)\right\|^{2} \leqslant \sigma_{n}$, где $\sigma_{n}$ - некоторая зависящая от коэффициентов и от начального условия убывающая к нулю последовательность («скорость сходимости»). Но, к сожалению, такая зависимость в этом случае далеко не очевидна даже при линейной зависимости коэффициентов от $x$.

Следствие 2.1. Предположим, что выполнены условия (2.2). Тогда конечномерные аппроксимачии $X_{n}(t)$, заданные формулой (2.3), сходятся к решению $X(t)$ уравнения (2.1) в среднем квадратическом, т.е. равномерно по $t \in[0, T]$

$$
\mathbf{E}\left\|X(t)-X_{n}(t)\right\|^{2} \longrightarrow 0, \quad n \rightarrow \infty .
$$

Д оказ ат е ль с т в о. Из тривиальных оценок $\left\|P_{n} a\right\| \leqslant\|a\|$ и $\left\|P_{n} b P_{n}\right\|_{\mathscr{L}_{2}} \leqslant\|b\|_{\mathscr{L}_{2}}$ следует справедливость условий (2.4) для коэффициентов уравнения (2.3). Условия (2.5) выполнены очевидным образом. Следовательно, мы можем применить теорему 2.1. Следствие 2.1 доказано.

Рассмотрим частный случай, когда коэффициенты $a, b$ не зависят от $t$.

Следствие 2.2. Если коэффициенты уравнений

$$
\begin{aligned}
X(t) & =X_{0}+\int_{0}^{t} a(s, X(s)) d s+\int_{0}^{t} b(s, X(s)) d W(s), \\
X_{n}(t) & =X_{0}^{n}+\int_{0}^{t} a_{n}\left(s, X_{n}(s)\right) d s+\int_{0}^{t} b_{n}\left(s, X_{n}(s)\right) d W(s)
\end{aligned}
$$

удовлетворяют условиям липшицевости и линейного роста (1.10a) $c$ общей для всех уравнений константой и для $s \in[0, T], x \in X$ $\left\|a_{n}(s, x)-a(s, x)\right\|+\left\|b_{n}(s, x)-b(s, x)\right\|_{\mathscr{L}_{2}}+\mathbf{E}\left\|X_{0}^{n}-X_{0}\right\|^{2} \longrightarrow 0, \quad n \rightarrow \infty$, то имеет место (равномерная по $t \in[0, T]$ ) сходимость

$$
\mathbf{E}\left\|X_{n}(t)-X(t)\right\|^{2} \longrightarrow 0, \quad n \rightarrow \infty \text {. }
$$

$B$ частности, конечномерные аппроксимачии

$$
Y_{n}(t)=P_{n} X_{0}+\int_{0}^{t} P_{n} a\left(s, Y_{n}(s)\right) d s+\int_{0}^{t} P_{n} b\left(s, Y_{n}(s)\right) P_{n} d W(s)
$$

сходятся к решению $X(t)$ в среднем квадратическом.

Рассмотрим теперь уравнение (1.1) и предположим, что выполнены условия (A)-(C) из п. 1.2. Предположим также, что $E_{n} \subset D(A)$. (Это выполняется, например, когда $A$ - дифференциальный оператор, а $E_{n}$ пространство многочленов.) Тогда оператор $A_{n}=P_{n} A P_{n}$ (как ограниченный) также порождает сильно непрерывную полугруппу. Конечномерные аппроксимации решений уравнения (1.1) определим как решения 
уравнения

$X_{n}(t)=P_{n} X_{0}+\int_{0}^{t}\left(P_{n} A X(s)+P_{n} a(s, X(s))\right) d s+\int_{0}^{t} P_{n} b(s, X(s)) P_{n} d W(s)$, или

$$
X_{n}(t)=U_{n}(t) X_{0}+\int_{0}^{t} U_{n}(t-s)\left(P_{n} a\left(s, X_{n}(s)\right) d s+P_{n} b\left(s, X_{n}(s)\right) P_{n} d W(s)\right)
$$

где $U_{n}(t)=e^{A_{n} t}$. Понятно, что ввиду ограниченности оператора $A_{n}$ для этого уравнения также выполнены условия $(\mathrm{A})-(\mathrm{C})$, гарантирующие существование и единственность решения. Проверка условий $(2.2)$ и (2.4a) не представляет сложностей. Первое из условий $(2.4 \mathrm{~b})$ перепишем следуюшим образом:

$$
\begin{aligned}
& \left\|\int_{s}^{t} U_{n}(v-u) A_{n} P_{n} a(u, x) d v\right\|+\left\|\int_{s}^{t} U_{n}(v-u) A_{n} P_{n} b(u, x) P_{n} d v\right\|_{\mathscr{L}_{2}} \\
& \quad \leqslant h(t-s)(1+\|x\|) .
\end{aligned}
$$

Это выполнено, например, когда подынтегральные выражения имеют линейный рост, т.е. когда

$$
\left\|U_{n}(v-u) A_{n} P_{n} a(u, x)\right\|+\left\|U_{n}(v-u) A_{n} P_{n} b(u, x) P_{n}\right\|_{\mathscr{L}_{2}} \leqslant C_{n}(1+\|x\|) .
$$

Если, допустим, $A P_{n}=P_{n} A$ (например, если $\left\{e_{n}\right\}-$ собственный базис оператора $A$ ), то проблем не возникает, выражение в левой части равняется

$$
\left\|P_{n} U(v-u) A a(u, x)\right\|+\left\|P_{n} U(v-u) A b(u, x) P_{n}\right\|_{\mathscr{L}_{2}}
$$

и оценивается сверху функцией $C\left(\|A a(u, x)\|+\|A b(u, x)\|_{\mathscr{L}_{2}}\right)$, имеющей линейный рост в силу условия (C).

Другой путь аппроксимации решения (1.1) состоит в следующем. Если оператор $A$ непрерывен, то по следствию 2.2 из теоремы 2.1 не нужно никаких дополнительных ограничений, чтобы получить сходимость. Таким образом, научившись приближать решения уравнения (1.1) решениями уравнения с непрерывным оператором в правой части, мы сумеем приблизить решения (1.1) конечномерными процессами. Этому вопросу посвящен следующий раздел.

\section{3. Аппроксимация решениями СДУ с ограниченными коэффициентами}

В этом разделе мы рассмотрим аппроксимации решений уравнения (1.1) решениями уравнения с ограниченными коэффициентами. 
Предположим, что коэффициенты уравнения (1.1) удовлетворяют условиям (A)-(С). Для $h>0$ положим $A_{h}=h^{-1}(U(h)-I) \in \mathscr{L}$ и рассмотрим уравнение

$$
X^{(h)}(t)=X_{0}+\int_{0}^{t}\left(A_{h} X^{(h)}(s)+a\left(s, X^{(h)}(s)\right)\right) d s+\int_{0}^{t} b\left(s, X^{(h)}(s)\right) d W(s) .
$$

Благодаря липшицевости и линейному росту коэффициентов $a, b$, a также ограниченности оператора $A$ существует единственное решение этого уравнения, которое также является мягким решением, т.е. решением уравнения

$X^{(h)}(t)=U^{(h)}(t) X_{0}+\int_{0}^{t} U^{(h)}(t-s)\left(a\left(s, X^{(h)}(s)\right) d s+b\left(s, X^{(h)}(s)\right) d W(s)\right)$,

где $U^{(h)}(t)=e^{A_{h} t}$. Предположим также, что $\mathbf{E}\left\|A X_{0}\right\|^{2}<\infty$. Как было отмечено ранее, из этого следует, что $\sup _{t \in[0, T]}\|A X(t)\|^{2}<\infty$. Справедлива следующая теорема.

Теорема 3.1. Если коэффиииенть уравнения (1.1) удовлетворяют условиям (A)-(C), то аппроксимачии $X^{(h)}$ сходятся $\kappa$ решению $X(t)$ этого уравнения, причем

$$
\mathbf{E}\left\|X(t)-X^{(h)}(t)\right\|^{2} \leqslant K h^{2 / 3}
$$

с константой $K$, не зависящей от $h$.

Д о к а з а т е л с т в о. Известно (см. [1]), что

$$
\left\|U(t) x-U^{(h)}(t) x\right\| \leqslant \omega_{T}\left(h^{1 / 3}, x\right)+C h^{1 / 3}\|x\|
$$

с константой, не зависящей от $h$. Здесь $\omega_{T}$ - модуль непрерывности полугруппы $U(t)$ :

$\omega_{T}(\varepsilon, x)=\sup \{\|U(t) x-U(s) x\|, 0 \leqslant s \leqslant t \leqslant T,|t-s|<\varepsilon\} \leqslant C \varepsilon\|A x\|$.

Из (3.2), в частности, следует, что нормы $\left\|U^{(h)}(t)\right\|$ ограничены равномерно по $h$ и $t \in[0, T]$. Оценим теперь $Z^{(h)}(t)$ :

$$
Z^{(h)}(t)=\mathbf{E}\left\|X(t)-X^{(h)}(t)\right\|^{2} \leqslant C\left(D_{1}+D_{2}+B_{1}+B_{2}\right),
$$

где

$$
\begin{aligned}
D_{1}= & \mathbf{E}\left\|\left(U(t)-U^{(h)}(t)\right) X_{0}\right\|^{2} \leqslant C \mathbf{E}\left(\omega_{T}^{2}\left(h^{1 / 3}, X_{0}\right)+h^{2 / 3}\left\|X_{0}\right\|^{2}\right) \\
\leqslant C h^{2 / 3}\left(\mathbf{E}\left\|A X_{0}\right\|^{2}+\mathbf{E}\left\|X_{0}\right\|^{2}\right) \leqslant C h^{2 / 3} & \\
D_{2}=\mathbf{E} \| \int_{0}^{t} U^{(h)}(t-s)( & \left(a(s, X(s))-a\left(s, X^{(h)}(s)\right)\right) d s \\
& \left.+\left(b(s, X(s))-b\left(s, X^{(h)}(s)\right)\right) d W(s)\right) \|^{2}
\end{aligned}
$$




$$
\begin{aligned}
& \leqslant C \mathbf{E} \int_{0}^{t}\left(\left\|a(s, X(s))-a\left(s, X^{(h)}(s)\right)\right\|^{2}\right. \\
& \left.+\left\|b(s, X(s))-b\left(s, X^{(h)}(s)\right)\right\|_{\mathscr{L}_{2}}^{2}\right) d s \\
& \leqslant C \int_{0}^{t} \mathbf{E}\left\|X(s)-X^{(h)}(s)\right\|^{2} d s=C \int_{0}^{t} Z^{(h)}(s) d s, \\
& B_{1}=\mathbf{E}\left\|\int_{0}^{t}\left(U(t-s)-U^{(h)}(t-s)\right) a(s, X(s)) d s\right\|^{2} \\
& \leqslant \mathbf{E} \int_{0}^{t} C h^{2 / 3}\left(\|a(s, X(s))\|^{2}+\|A a(s, X(s))\|^{2}\right) d s \\
& \leqslant C h^{2 / 3} \int_{0}^{t} \mathbf{E}\left(1+\|X(s)\|^{2}\right) d s \leqslant C h^{2 / 3}, \\
& B_{2}=\mathbf{E}\left\|\int_{0}^{t}\left(U(t-s)-U^{(h)}(t-s)\right) b(s, X(s)) d W(s)\right\|^{2} \\
& =\mathbf{E} \int_{0}^{t}\left\|\left(U(t-s)-U^{(h)}(t-s)\right) b(s, X(s))\right\|_{\mathscr{L}_{2}}^{2} d s \\
& =\mathbf{E} \int_{0}^{t} \sum_{n=1}^{\infty}\left\|\left(U(t-s)-U^{(h)}(t-s)\right) b(s, X(s)) e_{n}\right\|^{2} d s \\
& \leqslant C \mathbf{E} \int_{0}^{t} \sum_{n=1}^{\infty} h^{2 / 3}\left(\left\|b(s, X(s)) e_{n}\right\|^{2}+\left\|A b(s, X(s)) e_{n}\right\|^{2}\right) d s \\
& =C h^{2 / 3} \mathbf{E} \int_{0}^{t}\left(\|b(s, X(s))\|_{\mathscr{L}_{2}}^{2}+\|A b(s, X(s))\|_{\mathscr{L}_{2}}^{2}\right) d s \\
& \leqslant C h^{2 / 3} \int_{0}^{t} \mathbf{E}\left(1+\|X(s)\|^{2}\right) d s \leqslant C h^{2 / 3} \text {. }
\end{aligned}
$$

Таким образом, мы получили оценку

$$
Z^{(h)}(t) \leqslant C\left(h^{2 / 3}+\int_{0}^{t} Z^{(h)}(s) d s\right),
$$

откуда с использованием леммы Гронуолла следует утверждение теоремы.

\section{СПИСОК ЛИТЕРАТУРЫ}

1. Butzer P. L., Berens H. Semi-groups of Operators and Approximation. New York: Springer-Verlag, 1967, $318 \mathrm{p}$.

2. El Boukfaoui Y., Erraoui $M$. Remarks on the existence and approximation for semilinear stochastic differential equations in Hilbert spaces. - Stochastic Anal. Appl., 2002, v. 20, № 3, p. 495-518.

3. Clark J.M.C., Cameron R.J. The maximum rate of convergence of discrete approximations for stochastic differential equations. - Lectures Notes in Control and Inform. Sci., 1980, v. 25, p. 162-171.

4. $D u$ Q., Zhang T. Numerical approximation of some linear stochastic partial differential equations driven by special additive noises. - SIAM J. Numer. Anal., 2002, v. 40, № 4, p. 1421-1445. 
5. Greksch W., Tudor C. Stochastic Evolution Equations: A Hilbert Space Approach. Akademie-Verlag, 1995, $178 \mathrm{p}$.

6. Gyöngy I., Krylov N. On the rate of convergence of splitting-up approximations for SPDEs. - Stochastic Inequalities and Applications. Basel: Birkhäuser, 2003, p. 301321.

7. Gyöngy I., Krylov N. On the splitting-up method and stochastic partial differential equations. - Ann. Probab., 2003, v. 31, № 2, p. 564-591.

8. Gyöngy I., Millet A. On discretization schemes for stochastic evolution equations. Potential Anal., 2005, v. 23, № 2, p. 99-134.

9. Hausenblas E. Numerical analysis of semilinear stochastic evolution equations in Banach spaces. - J. Comput. Appl. Math., 2002, v. 147, № 2, p. 485-516.

10. Hausenblas E. Approximation for semilinear stochastic evolution equations. Potential Anal., 2003, v. 18, № 2, p. 141-186.

11. Kolodii $A$. On convergence of approximations of Itô-Volterra equations. - Progr. Systems Control Theory, 1997, v. 23, p. 157-165.

12. Kloeden P.E., Platen E. Numerical Solution of Stochastic Differential Equations. Berlin: Springer-Verlag, 1992, 632 p.

13. Maruyama $G$. Continuous Markov processes and stochastic equations. - Rend. Circ. Mat. Palermo, 1955, v. 4, p. 48-90.

14. Millet A., Sanz-Solé M. Approximation and support theorem for a wave equation in two space dimensions. - Bernoulli, 2000, v. 6, № 5, p. 887-915.

15. Pettersson R., Signahl M. Numerical approximation for a white noise driven SPDE with locally bounded drift. - Potential Anal., 2005, v. 22, № 4, p. 375-393.

16. Roman $L$. On numerical solutions of stochastic differential equations. $\mathrm{PhD}$ Thesis. Minneapolis: University of Minnesota, 2000.

17. Schurz $H$. A brief introduction to numerical analysis of (O)SDEs without tears. Report № 1670. Minneapolis: IMA, Univ. of Minnesota, 1999.

18. Shardlow T. Weak convergence of a numerical method for a stochastic heat equation. - BIT, 2003, v. 43, № 1, p. 179-193.

19. Wagner W., Platen E. Approximation of Itô integral equations. Report of ZIMM of Academy of Sciences of GDR, Berlin, February, 1978.

20. Yan $Y$. Error analysis and smoothing properties of discretized deterministic and stochastic parabolic problem. PhD Thesis. Göteborg: Chalmers University of Technology and Göteborg University, 2003.

21. Далецкий Ю. Л., Фомин С. В. Меры и дифференциальные уравнения в бесконечномерных пространствах. М.: Наука, 1983, 383 с.

22. Кузнецов Д. Ф. Некоторые вопросы численного решения стохастических дифференциальных уравнений. СПб.: Изд-во Санкт-Петербургского техн. ун-та, 1998.

23. Мильштейн Г.Н. Приближенное интегрирование стохастических дифференциальных уравнений. - Теория вероятн. и ее примен., 1974, т. 19, в. 3, с. 583-588.

24. Мильштейн $\Gamma$. $Н$. Численное интегрирование стохастических дифференциальных уравнений. Свердловск: Изд-во Уральского ун-та, 1988.

25. Шевченко Г. М. Швидкість збіжності дискретних апроксимацій розв'язків стохастичних диференціальних рівнянь у гільбертовому просторі. - Tеор. Імові̄p. матем. стат., 2003, v. 69, p. 172-183.

Поступила в редакцию

29.IX.2003

Исправленный вариант

14.IV.2006 